\title{
Periodic solutions for a kind of higher-order neutral functional differential equation with variable parameter
}

Aijun Yang ${ }^{*}$ and Helin Wang*

\section{*Correspondence:} yangaij2004@163.com; wh1982032@163.com College of Science, Zhejiang University of Technology, Hangzhou, Zhejiang 310032, P.R. China

\begin{abstract}
In this paper, we consider a kind of higher-order neutral equation with distributed delay and variable parameter:

$(x(t)-p(t) x(t-\sigma))^{(n)}+f(x(t)) x^{\prime}(t)+g\left(\int_{-r}^{0} x(t+s) d \alpha(s)\right)=q(t)$. By using the classical coincidence degree theory of Mawhin, sufficient conditions for the existence of periodic solutions are established. Recent results in the literature are generalized and significantly improved. Furthermore, two examples are given to illustrate that the results are almost sharp.

MSC: 34K10; 30D05; 34B45

Keywords: periodic solution; higher-order; neutral functional differential equation; variable parameter
\end{abstract}

\section{Introduction}

This paper is devoted to the application of Mawhin's continuation theorem to investigate the existence of periodic solutions for the following equation:

$$
(x(t)-p(t) x(t-\sigma))^{(n)}+f(x(t)) x^{\prime}(t)+g\left(\int_{-r}^{0} x(t+s) d \alpha(s)\right)=q(t),
$$

where $p, q$ are continuous periodic functions with period $T>0, p \in C^{n}(\mathbb{R}, \mathbb{R})$ with $|p(t)| \neq$ $1, f, g \in C(\mathbb{R}, \mathbb{R}), r>0, n$ is a positive integer, $\sigma \in \mathbb{R}, \alpha:[-r, 0] \rightarrow \mathbb{R}_{+}$is a bounded variation function, $\bigvee_{-r}^{0}(\alpha)=1$ and $\alpha(0) \neq \alpha(-r)$, where $\bigvee_{-r}^{0}(\alpha)$ is the total variation of $\alpha(s)$ over $[-r, 0]$.

In recent years, the existence of periodic solution for functional differential equations has been studied extensively (see [1-7]). For example, in [1], the authors studied the following equation with a deviating argument:

$$
x^{\prime \prime}(t)+f(x(t)) x^{\prime}(t)+g(x(t-\tau(t, x(t))))=e(t) .
$$

Du et al. [2] studied the second-order neutral equation with variable parameter:

$$
(x(t)-c(t) x(t-\tau))^{\prime \prime}+f(x(t)) x^{\prime}(t)+g(x(t-\gamma(t)))=e(t) .
$$

O2014 Yang and Wang; licensee Springer. This is an Open Access article distributed under the terms of the Creative Commons Attribution License (http://creativecommons.org/licenses/by/2.0), which permits unrestricted use, distribution, and reproduction in any medium, provided the original work is properly cited. 
In [2], the authors proved for the first time the lemma (Lemma 2.1) for the existence of $A^{-1}$ with $(A x)(t)=x(t)-c(t) x(t-\tau)$ and some properties of $A^{-1}$ when $c(t)$ is not a constant. Then they established sufficient conditions for the existence of periodic solutions of (1.3) by using Mawhin's theorem.

In [3], Wang and Lu discussed a kind of high-order neutral functional differential equation with distributed delay:

$$
(x(t)-c x(t-\sigma))^{(n)}+f(x(t)) x^{\prime}(t)+g\left(\int_{-r}^{0} x(t+s) d \alpha(s)\right)=p(t)
$$

with $|c| \neq 1$ a constant. However, there are several errors in the proof of Theorems 3.1 and 3.2 of [3]. The main purpose of this paper is to improve the results of [3] and modify the errors. Meanwhile, the problem considered in paper [2] is generalized to the higherorder case in our work. Moreover, two examples are given to demonstrate our results.

\section{Related lemmas}

For convenience we denote $p_{i}=\max _{t \in[0, T]}\left|p^{(i)}(t)\right|, \quad i=0,1, \ldots, n, p^{(0)}(t)=p(t), \quad \tau=$ $\min _{t \in[0, T]}|p(t)|$, and define the spaces

$$
C_{T}=\{x \in C(\mathbb{R}, \mathbb{R}): x(t+T) \equiv x(t), \forall t \in \mathbb{R}\}
$$

with the norm $|x|_{0}=\max _{t \in[0, T]}|x(t)|$ and

$$
C_{T}^{1}=\left\{x \in C^{1}(\mathbb{R}, \mathbb{R}): x(t+T) \equiv x(t), \forall t \in \mathbb{R}\right\}
$$

with the norm $\|x\|=\max \left\{|x|_{0},\left|x^{\prime}\right|_{0}\right\}$. Clearly, $C_{T}$ and $C_{T}^{1}$ are all Banach spaces.

Define a linear operator $A: C_{T} \rightarrow C_{T}$ by

$$
(A x)(t)=x(t)-p(t) x(t-\sigma), \quad \forall t \in[0, T] .
$$

Lemma 2.1 [2] If $|p(t)| \neq 1$, then $A$ has a continuous inverse $A^{-1}$ on $C_{T}$ satisfying

$$
\begin{aligned}
& \text { (1) }\left(A^{-1} x\right)(t)= \begin{cases}x(t)+\sum_{j=1}^{\infty} \prod_{i=1}^{j} p(t-(i-1) \sigma) x(t-j \sigma), & p_{0}<1, \forall x \in C_{T}, \\
-\frac{x(t+\sigma)}{p(t+\sigma)}-\sum_{j=1}^{\infty} \prod_{i=1}^{j+1} \frac{1}{p(t+i \sigma)} x(t+(j+1) \sigma), & \tau>1, \forall x \in C_{T} ;\end{cases} \\
& \text { (2) }\left|\left(A^{-1} x\right)(t)\right| \leq \begin{cases}\frac{|x|_{0}}{1-p_{0}}, & p_{0}<1, \forall x \in C_{T}, \\
\frac{|x| 0}{\tau-1}, & \tau>1, \forall x \in C_{T} ;\end{cases} \\
& \text { (3) } \int_{0}^{T}\left|\left(A^{-1} x\right)(t)\right| d t \leq \begin{cases}\frac{1}{1-p_{0}} \int_{0}^{T}|x(t)| d t, & p_{0}<1, \forall x \in C_{T}, \\
\frac{1}{\tau-1} \int_{0}^{T}|x(t)| d t, & \tau>1, \forall x \in C_{T} .\end{cases}
\end{aligned}
$$

Let $X$ and $Y$ be real Banach spaces and $L: \operatorname{dom} L \subset X \rightarrow Y$ be a Fredholm operator with index zero, i.e., $\operatorname{Im} L$ is closed and $\operatorname{dim} \operatorname{ker} L=\operatorname{codim} \operatorname{Im} L<\infty$. If $L$ is a Fredholm operator with index zero, then there exist continuous projections $P: X \rightarrow X$ and $Q: Y \rightarrow Y$ such that $\operatorname{Im} P=\operatorname{ker} L, \operatorname{Im} L=\operatorname{ker} Q$ and $L_{P}=\left.L\right|_{\operatorname{dom} L \cap \operatorname{ker} P}:(I-P) X \rightarrow \operatorname{Im} L$ is invertible. Denote by $K_{P}$ the inverse of $L_{P}$. Define $N: \bar{\Omega} \rightarrow X$, where $\Omega \subset X$ is an open and bounded set; $N$ is $L$-compact on $\bar{\Omega}$, if $Q N$ is continuous and bounded and $K_{P}(I-Q) N$ is compact on $\bar{\Omega}$. 
Lemma 2.2 [8] Let $X$ and $Y$ be two Banach spaces with norms $\|\cdot\|_{X}$ and $\|\cdot\|_{Y}$, respectively, and $\Omega \subset X$ an open and bounded set. Suppose $L: X \cap \operatorname{dom} L \rightarrow Y$ is a Fredholm operator of index zero and $N: \bar{\Omega} \rightarrow Y$ is L-compact. In addition, if

(i) $L x \neq \lambda N x$ for $\lambda \in(0,1), x \in(\operatorname{dom} L \backslash \operatorname{ker} L) \cap \partial \Omega$;

(ii) $N x \notin \operatorname{Im} L$ for $x \in \operatorname{ker} L \cap \partial \Omega$;

(iii) $\operatorname{deg}\left\{\left.J Q N\right|_{\bar{\Omega} \cap \operatorname{ker} L}, \Omega \cap \operatorname{ker} L, 0\right\} \neq 0$, where $J: \operatorname{Im} Q \rightarrow \operatorname{ker} L$ is a homeomorphism.

Then the abstract equation $L x=N x$ has at least one solution in $\bar{\Omega}$.

Define the linear operator $L: \operatorname{dom} L \subset C_{T}^{1} \rightarrow C_{T}$ by $L x=(A x)^{(n)}$ with

$$
\operatorname{dom} L=\left\{x \in C^{n}(\mathbb{R}, \mathbb{R}): x(t+T) \equiv x(t), t \in[0, T]\right\}=: C_{T}^{n}
$$

and the nonlinear operator

$$
N: C_{T}^{1} \rightarrow C_{T}, \quad(N x)(t)=-f(x(t)) x^{\prime}(t)-g\left(\int_{-r}^{0} x(t+s) d \alpha(s)\right)+q(t) .
$$

From $x \in \operatorname{ker} L,(x(t)-p(t) x(t-\sigma))^{(n)}=0$, we can obtain

$$
x(t)-p(t) x(t-\sigma)=c_{n-1} t^{n-1}+\cdots+c_{1} t+c_{0},
$$

where $c_{i} \in \mathbb{R}, i=0,1,2, \ldots, n-1$. From $x(t)-p(t) x(t-\sigma) \in C_{T}^{n}$, we have $c_{j}=0, j=1,2, \ldots$, $n-1$. Let $\omega \in C_{T}$ be a solution of $x(t)-p(t) x(t-\sigma)=1$, from Lemma 2.1, $\omega(t) \neq 0$, then

$$
\operatorname{ker} L=\{x \in \operatorname{dom} L: x(t)=c \omega(t), t \in[0, T]\} .
$$

Clearly, $\operatorname{Im} L=\left\{y \in C_{T}: \int_{0}^{T} y(t) d t=0\right\}$. Define continuous projections

$$
\begin{aligned}
& P: C_{T}^{1} \rightarrow C_{T}^{1}, \quad(P x)(t)=\frac{\int_{0}^{T} x(s) d s}{\int_{0}^{T} \omega(s) d s} \omega(t), \quad t \in[0, T], \\
& Q: C_{T} \rightarrow C_{T}, \quad(Q x)(t)=\frac{1}{T} \int_{0}^{T} y(s) d s, \quad t \in[0, T] .
\end{aligned}
$$

It is easy to see that $\operatorname{Im} P=\operatorname{ker} L$ and $\operatorname{ker} Q=\operatorname{Im} L . \operatorname{So} \operatorname{dim} \operatorname{ker} L=1=\operatorname{dim} \operatorname{Im} Q=\operatorname{codim} \operatorname{Im} L$. Notice that $\operatorname{Im} L$ is closed, then $L$ is a Fredholm operator of index zero.

Set $K_{P}=L_{P}^{-1}: \operatorname{Im} L \rightarrow \operatorname{dom} L \cap \operatorname{ker} P$ by

$$
\left(K_{P} y\right)(t)=A^{-1}\left\{\sum_{i=0}^{n-1} \frac{1}{i !}(A x)^{(i)}(0) t^{i}+\frac{1}{(n-1) !} \int_{0}^{t}(t-s)^{n-1} y(s) d s\right\}, \quad \forall t \in[0, T],
$$

where $(A x)^{(i)}(0), i=1,2, \ldots, n-1$, are defined by the equation $C X=B$; here

$$
\begin{aligned}
& X^{T}=\left((A x)^{(n-1)}(0),(A x)^{(n-2)}(0), \ldots,(A x)^{\prime}(0)\right) \\
& B^{T}=\left(b_{1}, b_{2}, \ldots, b_{n-1}\right), \quad b_{i}=-\frac{1}{i ! T} \int_{0}^{T}(T-s)^{i} y(s) d s
\end{aligned}
$$




$$
C=\left(\begin{array}{cccccc}
1 & 0 & 0 & \cdots & 0 & 0 \\
c_{1} & 1 & 0 & \cdots & 0 & 0 \\
c_{2} & c_{1} & 1 & \cdots & 0 & 0 \\
\vdots & \vdots & \vdots & \ddots & \vdots & \vdots \\
c_{n-3} & c_{n-4} & c_{n-5} & \cdots & 1 & 0 \\
c_{n-2} & c_{n-3} & c_{n-4} & \cdots & c_{1} & 1
\end{array}\right)_{(n-1) \times(n-1)}
$$

with

$$
c_{j}=\frac{T^{j}}{(j+1) !}, \quad j=1,2, \ldots, n-2 .
$$

Whereafter, since $\operatorname{ker} P=\left\{x \in C_{T}^{1}: \int_{0}^{T} x(s) d s=0\right\},(A x)(0)$ can be determined by

$$
\int_{0}^{T} A^{-1}\left\{\sum_{i=0}^{n-1} \frac{1}{i !}(A x)^{(i)}(0) t^{i}+\frac{1}{(n-1) !} \int_{0}^{t}(t-s)^{n-1} y(s) d s\right\} d t=0 .
$$

It follows from Lemma 2.1, the definition of $N, Q, K_{P}$, and the continuity of $f, g, q$ that $N$ is $L$-compact.

\section{Main results}

Theorem 3.1 Suppose $n$ is an even integer and $\sum_{i=0}^{k} C_{k}^{i} p_{i} T^{i}<1$ with $k=\frac{n}{2}$. In addition, if there exist constants $a \geq 0$ and $M>0$ such that

(H1) $x \cdot(g(x)-\bar{q})>0($ or $<0)$, whenever $|x|>M$, where $\bar{q}=\frac{1}{T} \int_{0}^{T} q(t) d t$,

(H2) $\lim \sup _{|x| \rightarrow \infty} \max _{t \in[0, T]} \frac{|g(x)-q(t)|}{|x|} \leq a$,

then (1.1) has at least one periodic solution provided

$$
\frac{a T^{n}}{1-\sum_{i=0}^{k} C_{k}^{i} p_{i} T^{i}}<1
$$

Proof Without loss of generality, we may assume that $x \cdot(g(x)-\bar{q})>0$, when $|x|>M$. Now, we will complete the proof by three steps.

Step 1. Let $\Omega_{1}=\{x \in \operatorname{dom} L \backslash \operatorname{ker} L: L x=\lambda N x, \lambda \in(0,1)\}, \forall x \in \Omega_{1}, L x=\lambda N x$, i.e.,

$$
(x(t)-p(t) x(t-\sigma))^{(n)}+\lambda f(x(t)) x^{\prime}(t)+\lambda g\left(\int_{-r}^{0} x(t+s) d \alpha(s)\right)=\lambda q(t) .
$$

Integrating both sides of (3.2) on $[0, T]$, we have

$$
\int_{0}^{T}\left[g\left(\int_{-r}^{0} x(t+s) d \alpha(s)\right)-\bar{q}\right] d t=0 .
$$

By the integral mean value theorem, there exists a constant $\xi \in(0, T)$ such that $g\left(\int_{-r}^{0} x(\xi+\right.$ $s) d \alpha(s))-\bar{q}=0$. So (H1) implies that $\left|\int_{-r}^{0} x(\xi+s) d \alpha(s)\right| \leq M$. Since $\bigvee_{-r}^{0}(\alpha)=1$ and $\alpha(-r) \neq \alpha(0)$ on $t \in[-r, 0]$, by the properties of the Riemann-Stieltjes integral, there exists a constant $\eta \in(-r, 0)$ such that $|x(\xi+\eta)| \leq M$. Because $x(t)$ is a $T$-periodic function, there exists an integer $k_{0}$ such that $\xi+\eta=k_{0} T+t^{*}, t^{*} \in[0, T)$. Then $\left|x\left(t^{*}\right)\right| \leq M$. Hence,

$$
|x|_{0}=\max _{t \in[0, T]}\left|x\left(t^{*}\right)+\int_{t^{*}}^{t} x^{\prime}(t) d t\right| \leq M+\int_{0}^{T}\left|x^{\prime}(t)\right| d t .
$$


Yang and Wang Advances in Difference Equations 2014, 2014:187

Page 5 of 10

Multiplying both sides of (3.2) by $x(t)$ and integrating them over [0,T], one gets

$$
\begin{aligned}
(-1)^{k} \int_{0}^{T}\left|x^{(k)}(t)\right|^{2} d t & \\
= & (-1)^{k} \int_{0}^{T}(p(t) x(t-\sigma))^{(k)} x^{(k)}(t) d t-\lambda \int_{0}^{T}\left[g\left(\int_{-r}^{0} x(t+s) d \alpha(s)\right)-q(t)\right] x(t) d t \\
= & (-1)^{k} \sum_{i=0}^{k} C_{k}^{i} \int_{0}^{T} p^{(i)}(t) x^{(k-i)}(t-\sigma) x^{(k)}(t) d t \\
& -\lambda \int_{0}^{T}\left[g\left(\int_{-r}^{0} x(t+s) d \alpha(s)\right)-q(t)\right] x(t) d t
\end{aligned}
$$

Since $x(0)=x(T), x^{\prime}(0)=x^{\prime}(T), \ldots, x^{(n-1)}(0)=x^{(n-1)}(T)$, there exist $\xi_{i} \in(0, T), i=1,2, \ldots, n$, such that $x^{\prime}\left(\xi_{1}\right)=x^{\prime \prime}\left(\xi_{2}\right)=\cdots=x^{(n)}\left(\xi_{n}\right)=0$. Thus,

$$
\int_{0}^{T}\left|x^{\prime}(t)\right| d t \leq T \int_{0}^{T}\left|x^{\prime \prime}(t)\right| d t \leq \cdots \leq T^{n-1} \int_{0}^{T}\left|x^{(n)}(t)\right| d t
$$

and

$$
\int_{0}^{T}\left|x^{\prime}(t)\right|^{2} d t \leq T^{2} \int_{0}^{T}\left|x^{\prime \prime}(t)\right|^{2} d t \leq \cdots \leq T^{2(n-1)} \int_{0}^{T}\left|x^{(n)}(t)\right|^{2} d t
$$

By the Hölder inequality and (3.7), we have

$$
\begin{aligned}
\int_{0}^{T}\left|x^{(k)}(t)\right|^{2} d t \leq & \sum_{i=0}^{k} C_{k}^{i} p_{i}\left(\int_{0}^{T}\left|x^{(k-i)}(t-\sigma)\right|^{2} d t\right)^{\frac{1}{2}}\left(\int_{0}^{T}\left|x^{(k)}(t)\right|^{2} d t\right)^{\frac{1}{2}} \\
& +|x|_{0} \int_{0}^{T}\left|g\left(\int_{-r}^{0} x(t+s) d \alpha(s)\right)-q(t)\right| d t \\
\leq & \sum_{i=0}^{k} C_{k}^{i} p_{i} T^{i} \int_{0}^{T}\left|x^{(k)}(t)\right|^{2} d t \\
& +|x|_{0} \int_{0}^{T}\left|g\left(\int_{-r}^{0} x(t+s) d \alpha(s)\right)-q(t)\right| d t
\end{aligned}
$$

In view of $(\mathrm{H} 2)$ and the properties of the bounded variation function, $\forall \varepsilon>0$, there exists a constant $\rho>M$ such that

$$
\left|g\left(\int_{-r}^{0} x(t+s) d \alpha(s)\right)-q(t)\right| \leq(a+\varepsilon)\left|\int_{-r}^{0} x(t+s) d \alpha(s)\right| \leq(a+\varepsilon)|x|_{0}
$$

when $\left|\int_{-r}^{0} x(t+s) d \alpha(s)\right|>\rho$. Let

$$
\begin{aligned}
& E_{1}=\left\{t \in[0, T]:\left|\int_{-r}^{0} x(t+s) d \alpha(s)\right|>\rho\right\}, \\
& E_{2}=\left\{t \in[0, T]:\left|\int_{-r}^{0} x(t+s) d \alpha(s)\right| \leq \rho\right\} .
\end{aligned}
$$


Hence,

$$
\begin{aligned}
\int_{0}^{T}\left|g\left(\int_{-r}^{0} x(t+s) d \alpha(s)\right)-q(t)\right| d t & =\left(\int_{E_{1}}+\int_{E_{2}}\right)\left|g\left(\int_{-r}^{0} x(t+s) d \alpha(s)\right)-q(t)\right| d t \\
& \leq T(a+\varepsilon)|x|_{0}+T \tilde{g}_{\rho}
\end{aligned}
$$

where $\tilde{g}_{\rho}=\max _{t \in E_{2}}\left|g\left(\int_{-r}^{0} x(t+s) d \alpha(s)\right)-q(t)\right|$. By (3.4) and (3.6), we get

$$
\begin{aligned}
|x|_{0} & \leq M+\int_{0}^{T}\left|x^{\prime}(t)\right| d t \leq M+T^{k-1} \int_{0}^{T}\left|x^{(k)}(t)\right| d t \\
|x|_{0}^{2} & \leq M^{2}+2 M T^{k-1} \int_{0}^{T}\left|x^{(k)}(t)\right| d t+T^{2(k-1)}\left(\int_{0}^{T}\left|x^{(k)}(t)\right| d t\right)^{2} \\
& \leq M^{2}+2 M T^{k-1} \int_{0}^{T}\left|x^{(k)}(t)\right| d t+T^{2 k-1} \int_{0}^{T}\left|x^{(k)}(t)\right|^{2} d t .
\end{aligned}
$$

Thus,

$$
\begin{aligned}
\int_{0}^{T}\left|x^{(k)}(t)\right|^{2} d t \leq & \frac{1}{1-\sum_{i=0}^{k} C_{k}^{i} p_{i} T^{i}}\left[T(a+\varepsilon)|x|_{0}^{2}+T \tilde{g}_{\rho}|x|_{0}\right] \\
\leq & \frac{1}{1-\sum_{i=0}^{k} C_{k}^{i} p_{i} T^{i}}\left[(a+\varepsilon) T^{2 k} \int_{0}^{T}\left|x^{(k)}(t)\right|^{2} d t\right. \\
& \left.+\left(2(a+\varepsilon) M T^{k}+T^{k} \tilde{g}_{\rho}\right) \int_{0}^{T}\left|x^{(k)}(t)\right| d t+(a+\varepsilon) T M^{2}+M T \tilde{g}_{\rho}\right] \\
\leq & \frac{1}{1-\sum_{i=0}^{k} C_{k}^{i} p_{i} T^{i}}\left[(a+\varepsilon) T^{n} \int_{0}^{T}\left|x^{(k)}(t)\right|^{2} d t\right. \\
& +T^{k+\frac{1}{2}}\left(2(a+\varepsilon) M+\tilde{g}_{\rho}\right)\left(\int_{0}^{T}\left|x^{(k)}(t)\right|^{2} d t\right)^{\frac{1}{2}} \\
& \left.+(a+\varepsilon) T M^{2}+M T \tilde{g}_{\rho}\right]
\end{aligned}
$$

In view of $\frac{a T^{n}}{1-\sum_{i=0}^{k} C_{k}^{i} p_{i} T^{i}}<1$, there exists a constant $M_{1}>0$ such that $\int_{0}^{T}\left|x^{(k)}(t)\right|^{2} d t \leq M_{1}$.

Then

$$
\begin{aligned}
& |x|_{0} \leq M+T^{k-1} \int_{0}^{T}\left|x^{(k)}(t)\right| d t \leq M+T^{k-\frac{1}{2}} M_{1}^{\frac{1}{2}}=: M_{2}, \\
& \left|x^{\prime}\right|_{0} \leq \int_{0}^{T}\left|x^{\prime \prime}(t)\right| d t \leq T^{k-2} \int_{0}^{T}\left|x^{(k)}(t)\right| d t \leq T^{k-\frac{3}{2}} M_{1}^{\frac{1}{2}}=: M_{3} .
\end{aligned}
$$

Step 2. Let $\Omega_{2}=\{x \in \operatorname{ker} L: N x \in \operatorname{Im} L\}$. Then for $x \in \Omega_{2}, x(t)=c \omega(t), c \in \mathbb{R}$, and $N x \in$ $\operatorname{Im} L=\operatorname{ker} Q$, that is,

$$
Q N x=-\frac{1}{T} \int_{0}^{T}\left[g\left(\int_{-r}^{0} c \omega(t+s) d \alpha(s)\right)-\bar{q}\right] d t=0 .
$$

Repeating the process of Step 1 , we see that $\Omega_{2}$ is bounded, that is, there exists $M_{4}>0$ such that $\|x\| \leq M_{4}$ for $x \in \Omega_{2}$. 
Step 3. Let $\Omega=\{x \in \operatorname{dom} L:\|x\|<\widehat{M}\}$, where $\widehat{M}=\max \left\{M_{2}, M_{3}, M_{4}\right\}+1$, then $\bar{\Omega}_{1} \cup$ $\bar{\Omega}_{2} \subset \Omega$. In view of Step 1 and Step 2, conditions (i) and (ii) in Lemma 2.2 are all satisfied. Next, we will show that (iii) of Lemma 2.2 holds.

Set the isomorphism $J: \operatorname{Im} Q \rightarrow \operatorname{ker} L$ by $J(x)=-x$. For $x \in \bar{\Omega} \cap \operatorname{ker} L, \mu \in[0,1]$, define the homotopy

$$
H(x, \mu)=\mu x+(1-\mu) J Q N x,
$$

i.e.,

$$
H(x, \mu)=\mu x+\frac{1-\mu}{T} \int_{0}^{T}\left[g\left(\int_{-r}^{0} x(t+s) d \alpha(s)\right)-\bar{q}\right] d t .
$$

Since $\|x\|>M$ for $x \in \partial \Omega \cap \operatorname{ker} L, x \cdot(g(x)-\bar{q})>0$. So $H(x, \mu) \neq 0$ for $(x, \mu) \in(\partial \Omega \cap \operatorname{ker} L) \times$ $[0,1]$. Hence, by the homotopy invariance of the Brouwer degree, we obtain

$$
\begin{aligned}
\operatorname{deg}\left\{\left.J Q N\right|_{\bar{\Omega} \cap \operatorname{ker} L}, \Omega \cap \operatorname{ker} L, 0\right\} & =\operatorname{deg}\{H(\cdot, 0), \Omega \cap \operatorname{ker} L, 0\} \\
& =\operatorname{deg}\{H(\cdot, 1), \Omega \cap \operatorname{ker} L, 0\} \\
& =\operatorname{deg}\{I, \Omega \cap \operatorname{ker} L, 0\} \neq 0 .
\end{aligned}
$$

Applying Lemma 2.2, we reach the conclusion.

For the case $x \cdot(g(x)-\bar{q})<0$, when $|x|>M$, a similar argument can complete the proof. Here we omit it.

Remark 3.1 In [3], the calculation of formula (3.5) is wrong. So the bound of $\int_{0}^{T} \mid g\left(\int_{-r}^{0} x(t+\right.$ s) $d \alpha(s))-q(t) \mid d t$ is not evaluated correctly. The same error appeared again in the next theorem. We modified those errors in this paper.

Theorem 3.2 Suppose that $n$ is an odd integer and $\sum_{i=0}^{k} C_{k}^{i} p_{i} T^{i}<1$, where $k=\frac{n+1}{2}$ and $k$ is odd. Moreover, iff $(y) \geq 0, \forall y \in \mathbb{R}$ and conditions (H1), (H2) and (3.1) are satisfied. Then (1.1) has at least one periodic solution.

Proof Without loss of generality, we assume that $x \cdot(g(x)-\bar{q})>0$, when $|x|>M$. As in the proof of Theorem 3.1, define the set $\Omega_{1}$, for $x \in \Omega_{1}, \lambda \in(0,1)$, (3.2) can be obtained. Multiplying both sides of (3.2) by $x^{\prime}(t)$ and integrating them over $[0, T]$, we obtain

$$
\begin{aligned}
(-1)^{k-1} \int_{0}^{T}\left|x^{(k)}(t)\right|^{2} d t= & (-1)^{k-1} \int_{0}^{T}(p(t) x(t-\sigma))^{(k)} x^{(k)}(t) d t-\lambda \int_{0}^{T} f(x(t))\left|x^{\prime}(t)\right|^{2} d t \\
& -\lambda \int_{0}^{T}\left[g\left(\int_{-r}^{0} x(t+s) d \alpha(s)\right)-q(t)\right] x^{\prime}(t) d t \\
\leq & (-1)^{k-1} \sum_{i=0}^{k} C_{k}^{i} \int_{0}^{T} p^{(i)}(t) x^{(k-i)}(t-\sigma) x^{(k)}(t) d t \\
& -\lambda \int_{0}^{T}\left[g\left(\int_{-r}^{0} x(t+s) d \alpha(s)\right)-q(t)\right] x^{\prime}(t) d t .
\end{aligned}
$$


In view of

$$
|x|_{0} \leq M+\int_{0}^{T}\left|x^{\prime}(t)\right| d t \leq M+T\left|x^{\prime}\right|_{0}
$$

by the Hölder inequality and (3.6), (3.7) in the proof of Theorem 3.1, one gets

$$
\begin{aligned}
& \int_{0}^{T}\left|x^{(k)}(t)\right|^{2} d t \leq \sum_{i=0}^{k} C_{k}^{i} p_{i}\left(\int_{0}^{T}\left|x^{(k-i)}(t-\sigma)\right|^{2} d t\right)^{\frac{1}{2}}\left(\int_{0}^{T}\left|x^{(k)}(t)\right|^{2} d t\right)^{\frac{1}{2}} \\
& +\left|x^{\prime}\right|_{0} \int_{0}^{T}\left|g\left(\int_{-r}^{0} x(t+s) d \alpha(s)\right)-q(t)\right| d t \\
& \leq \sum_{i=0}^{k} C_{k}^{i} p_{i} T^{i} \int_{0}^{T}\left|x^{(k)}(t)\right|^{2} d t \\
& +\left|x^{\prime}\right|_{0} \int_{0}^{T}\left|g\left(\int_{-r}^{0} x(t+s) d \alpha(s)\right)-q(t)\right| d t \\
& \leq \frac{1}{1-\sum_{i=0}^{k} C_{k}^{i} p_{i} T^{i}}\left|x^{\prime}\right|_{0} \int_{0}^{T}\left|g\left(\int_{-r}^{0} x(t+s) d \alpha(s)\right)-q(t)\right| d t \\
& \leq \frac{1}{1-\sum_{i=0}^{k} C_{k}^{i} p_{i} T^{i}}\left[(a+\varepsilon) T|x|_{0}\left|x^{\prime}\right|_{0}+T \tilde{g}_{\rho}\left|x^{\prime}\right|_{0}\right] \\
& \leq \frac{1}{1-\sum_{i=0}^{k} C_{k}^{i} p_{i} T^{i}}\left[(a+\varepsilon) T\left(M+T\left|x^{\prime}\right|_{0}\right)\left|x^{\prime}\right|_{0}+T \tilde{g}_{\rho}\left|x^{\prime}\right|_{0}\right] \\
& \leq \frac{1}{1-\sum_{i=0}^{k} C_{k}^{i} p_{i} T^{i}}\left[(a+\varepsilon) T^{2 k-1} \int_{0}^{T}\left|x^{(k)}(t)\right|^{2} d t\right. \\
& \left.+\left((a+\varepsilon) M+\tilde{g}_{\rho}\right) T^{k-1} \int_{0}^{T}\left|x^{(k)}(t)\right| d t\right] \\
& \leq \frac{1}{1-\sum_{i=0}^{k} C_{k}^{i} p_{i} T^{i}}\left[(a+\varepsilon) T^{n} \int_{0}^{T}\left|x^{(k)}(t)\right|^{2} d t\right. \\
& \left.+\left((a+\varepsilon) M+\tilde{g}_{\rho}\right) T^{k-\frac{1}{2}}\left(\int_{0}^{T}\left|x^{(k)}(t)\right|^{2} d t\right)^{\frac{1}{2}}\right] \text {. }
\end{aligned}
$$

Since the condition (3.1) holds, $\int_{0}^{T}\left|x^{(k)}(t)\right|^{2} d t$ is bounded. The remainder can be proved in the same way as in Theorem 3.1.

Remark 3.2 In [3], the first inequality in (3.17) cannot be obtained without assuming that $k=\frac{n+1}{2}$ is odd. In our paper, we correct this error by adding such a condition.

Remark 3.3 In our paper, we consider the $n$-order period equation (1.1); in this sense, we generalize the model in [2] to higher order under the equivalent conditions. Moreover, in view of the variable parameter $p(t)$ in (1.1), we develop the results in [3] with the constant coefficient $c$.

\section{Examples}

As an application, we list the following examples. 
Example 4.1 Consider

$$
\begin{aligned}
& \left(x(t)-\frac{1}{960} \sin t x(t-\sigma)\right)^{(6)}+2 e^{4\left(x^{2}(t)-1\right)} x^{\prime}(t) \\
& \quad+\int_{-\pi}^{0} x(t+s) d s \cdot e^{-\left(\int_{-\pi}^{0} x(t+s) d s\right)^{2}}=-\cos t+\frac{1}{30} \sin 2 t .
\end{aligned}
$$

Corresponding to (1.1), we have $p(t)=\frac{1}{960} \sin t, g(x)=x e^{-x^{2}}, f(x)=2 e^{4\left(x^{2}-1\right)}, q(t)=-\cos t+$ $\frac{1}{30} \sin 2 t, \sigma=2 \pi, r=\pi, n=6, k=3, T=2 \pi$. Obviously, $p_{i}=\frac{1}{960}, i=0,1,2, \ldots, 6$; we have

$$
\begin{aligned}
& \sum_{i=0}^{3} C_{3}^{i} \frac{1}{960}(2 \pi)^{i} \leq \frac{8 \pi^{3}+12 \pi^{2}+6 \pi+1}{960}<1, \\
& a=\lim _{|x| \rightarrow \infty} \max _{t \in[0,2 \pi]} \frac{|g(x)-q(t)|}{|x|}=0
\end{aligned}
$$

and

$$
\frac{a T^{2 k}}{1-\sum_{i=0}^{3} C_{3}^{i} \frac{1}{960}(2 \pi)^{i}}=0<1 .
$$

Therefore, Theorem 3.1 implies that (4.1) has at least one $2 \pi$-periodic solution. In fact, $x(t)=\cos t$ is such a solution.

Example 4.2 Consider

$$
\begin{aligned}
& \left(x(t)-\frac{1}{480} \sin t x(t-\sigma)\right)^{(5)}+2 e^{4\left(x^{2}(t)-1\right)} x^{\prime}(t) \\
& \quad+\int_{-\pi}^{0} x(t+s) d s \cdot e^{-\left(\int_{-\pi}^{0} x(t+s) d s\right)^{2}}=\cos t-\frac{1}{30} \sin 2 t .
\end{aligned}
$$

Corresponding to (1.1), we have $p(t)=\frac{1}{480} \sin t, g(x)=x e^{-x^{2}}, f(x)=2 e^{4\left(x^{2}-1\right)}, q(t)=\cos t-$ $\frac{1}{30} \sin 2 t, \sigma=2 \pi, r=\pi, n=5, k=3, T=2 \pi$. Obviously, $p_{i}=\frac{1}{480}, i=0,1,2, \ldots, 5$; we have

$$
\begin{aligned}
& \sum_{i=0}^{3} C_{3}^{i} \frac{1}{480}(2 \pi)^{i} \leq \frac{8 \pi^{3}+12 \pi^{2}+6 \pi+1}{480}<1, \\
& a=\lim _{|x| \rightarrow \infty} \max _{t \in[0,2 \pi]} \frac{|g(x)-q(t)|}{|x|}=0
\end{aligned}
$$

and

$$
\frac{a T^{2 k-1}}{1-\sum_{i=0}^{3} C_{3 \frac{1}{480}}^{i}(2 \pi)^{i}}=0<1 .
$$

Therefore, Theorem 3.2 implies that (4.2) has at least one $2 \pi$-periodic solution. It is easy to see that $x(t)=\sin t$ is such a solution. 
Authors' contributions

AY performed the theory analysis and carried out the computations. HW participated in the design of the study and helped to draft the manuscript. All authors have read and approved the final manuscript.

\section{Acknowledgements}

The work is supported by the National Natural Science Foundation of China (No. 11226148 and No. 61273016) and the Natural Science Foundation of Zhejiang Province (LY12F05006).

Received: 21 March 2014 Accepted: 26 June 2014 Published: 22 Jul 2014

\section{References}

1. Lu, S, Ge, W: Periodic solutions for a kind of Liénard equation with a deviating argument. J. Math. Anal. Appl. 289, 231-243 (2004)

2. Du, B, Guo, L, Ge, W, Lu, S: Periodic solutions for generalized Liénard neutral equation with variable parameter. Nonlinear Anal. 70, 2387-2394 (2009)

3. Wang, K, Lu, S: On the existence of periodic solutions for a kind of high-order neutral functional differential equation. J. Math. Anal. Appl. 326, 1161-1173 (2007)

4. Lu, S, Ge, W: Existence of periodic solutions for a kind of second order neutral functional differential equation. Appl. Math. Comput. 157, 433-448 (2004)

5. Candan, T: Existence of nonoscillatory solutions of first-order nonlinear neutral differential equations. Appl. Math. Lett. 26(12), 1182-1186 (2013)

6. Ma, S, Wang, Z, Yu, J: An abstract theorem at resonance and its applications. J. Differ. Equ. 145(2), 274-294 (1998)

7. Chalishajar, DN, Karthikeyan, K: Existence and uniqueness results for boundary value problems of higher order fractional integro-differential equations involving Gronwall's inequality in Banach spaces. Acta Math. Sci. 33(3), 758-772 (2013)

8. Gaines, R, Mawhin, J: Coincidence Degree and Nonlinear Differential Equations. Springer, Berlin (1977)

10.1186/1687-1847-2014-187

Cite this article as: Yang and Wang: Periodic solutions for a kind of higher-order neutral functional differential equation with variable parameter. Advances in Difference Equations 2014, 2014:187

\section{Submit your manuscript to a SpringerOpen ${ }^{\circ}$ journal and benefit from:}

- Convenient online submission

- Rigorous peer review

Immediate publication on acceptance

- Open access: articles freely available online

- High visibility within the field

- Retaining the copyright to your article 